\title{
Soluble HLA-G and HLA-E Levels in Bone Marrow Plasma Samples Are Related to Disease Stage in Neuroblastoma Patients
}

\author{
Fabio Morandi, ${ }^{1}$ Sarah Pozzi, ${ }^{2}$ Barbara Carlini, ${ }^{1}$ Loredana Amoroso, ${ }^{3}$ \\ Vito Pistoia, ${ }^{1}$ and Maria Valeria Corrias ${ }^{1}$ \\ ${ }^{1}$ Laboratorio di Oncologia, Istituto Giannina Gaslini, Via Gaslini 5, 16147 Genoa, Italy \\ ${ }^{2}$ Centro Cellule Staminali, IRCCS AOU San Martino-IST, Largo R. Benzi 10, 16132 Genoa, Italy \\ ${ }^{3}$ UOC Oncologia, Istituto Giannina Gaslini, Via Gaslini 5, 16147 Genoa, Italy
}

Correspondence should be addressed to Fabio Morandi; fabiomorandi@gaslini.org

Received 20 May 2016; Revised 4 July 2016; Accepted 19 July 2016

Academic Editor: Ban-Hock Toh

Copyright (C) 2016 Fabio Morandi et al. This is an open access article distributed under the Creative Commons Attribution License, which permits unrestricted use, distribution, and reproduction in any medium, provided the original work is properly cited.

\begin{abstract}
The role of nonclassical HLA-class Ib molecules HLA-G and HLA-E in the progression of Neuroblastoma (NB), the most common pediatric extracranial solid tumor, has been characterized in the last years. Since BM infiltration by NB cells is an adverse prognostic factor, we have here analyzed for the first time the concentration of soluble (s)HLA-G and HLA-E in bone marrow (BM) plasma samples from NB patients at diagnosis and healthy donors. sHLA-G and sHLA-E are present in BM plasma samples, and their levels were similar between NB patients and controls, thus suggesting that these molecules are physiologically released by resident or stromal BM cell populations. This hypothesis was supported by the finding that sHLA-G and sHLA-E levels did not correlate with $\mathrm{BM}$ infiltration and other adverse prognostic factors (MYCN amplification and age at diagnosis). In contrast, BM plasma levels of both molecules were higher in patients with metastatic disease than in patients with localized NB, thus suggesting that concentration of these molecules might be correlated with disease progression. The prognostic role of sHLA-G and sHLA-E concentration in the BM plasma for NB patients will be evaluated in future studies, by analyzing the clinical outcome of the same NB patients at followup.
\end{abstract}

\section{Introduction}

Neuroblastoma (NB) is the most common extracranial solid tumor in children, with an incidence of 1 case per 100.000 children per year, and causes $15 \%$ of cancer deaths in pediatric age. NB originates from the sympathetic nervous system, most frequently in the adrenal medulla or the paraspinal ganglia. The causes are unknown, although 1-2\% of NB may have a hereditary basis. Different genetic alterations have been characterized in NB, that is, gain-of-function of $A L K$ gene, losses of $11 \mathrm{q}$ and $1 \mathrm{p}$, gain of $17 \mathrm{q}$, and amplification of the MYCN gene. NB is heterogeneous, as it may undergo spontaneous remission or evolve to progressive metastatic disease, with dissemination to lymph nodes, bone, bone marrow, liver, skin, and other organs [1]. In particular, $\mathrm{BM}$ infiltration is an indicator of poor outcome for $\mathrm{NB}$ patients [2]. The International Neuroblastoma Risk Group staging system takes into account genetic alterations, DNA ploidy, histological features, and clinical data, as criteria for defining the risk classes. The prognosis of low/intermediate risk NB patients is favorable, and tumors can be cured by surgery alone or minimal chemotherapy. In contrast, highrisk NB patients' prognosis is poor, in spite of aggressive treatment based on surgery, chemotherapy, radiation therapy, hematopoietic stem cell transplantation, and adjuvant therapy with retinoic acid. In fact, survival rates of these patients at 5 years are less than $50 \%$ [3].

In the last years, the role of HLA-class Ib molecules in the progression of NB has been characterized by our group [47] and by others [8]. HLA-Ib family includes HLA-G, HLAE, HLA-F, and HLA-H. In contrast with high polymorphic HLA-Ia molecules (HLA-A, HLA-B, and HLA-C) all these molecules display a limited polymorphism, with few alleles encoding a limited number of functional proteins. Moreover, although HLA-class Ib molecules can bind small peptides and present them to specific $\mathrm{CD} 8^{+} \mathrm{T}$ cell subsets (similarly 
TABLE 1: Neuroblastoma patients' characteristics. First row indicates all the variables analyzed in NB patients, second row indicates the subgroups for each variable, and third row indicates the number of subjects in each group.

\begin{tabular}{lcccccccccccccc}
\hline \multicolumn{2}{c}{$\begin{array}{c}\text { Age at diagnosis } \\
\text { (months) }\end{array}$} & \multicolumn{2}{c}{ Sex } & \multicolumn{2}{c}{ MYCN } & \multicolumn{3}{c}{ BM infiltration } & \multicolumn{3}{c}{ Stage } \\
$<18$ & $>18$ & M & F & s.c. & Ampl. & Neg. & $1+$ & $2+$ & $3+$ & 1 & 2 & 3 & 4 & $4 s$ \\
\hline 19 & 12 & 12 & 19 & 22 & 9 & 19 & 5 & 4 & 3 & 6 & 1 & 10 & 12 & 2 \\
\hline
\end{tabular}

s.c.: single copy; Ampl.: amplified; Neg.: negative.

to HLA-class Ia counterparts), their main function is the modulation of the immune response in both physiological and pathological conditions [9]. HLA-G and HLA-E are the best characterized among HLA-Ib molecules. HLA-G has seven different isoforms, four membrane bound (namely, HLA-G1, HLA-G2, HLA-G3, and HLA-G4) and three soluble (namely, HLA-G5, HLA-G6, and HLA-G7), that are generated by alternative splicing from the same primary transcript. HLA-G can interact with at least four receptors, namely, immunoglobulin-like transcript (ILT)2, ILT4, KIR2DL4, and CD160, thus affecting the function of different immune effector cells ( $\mathrm{T}$ and B lymphocytes, natural killer NK cells, dendritic cells, granulocytes, and monocytes) [10]. In contrast, HLA-E can be expressed as membrane bound or soluble isoform (generated through metalloproteases cleavage) and can inhibit $\mathrm{CD}^{+} \mathrm{T}$ cells or NK cells though interaction with CD94/NKG2A heterodimeric receptor. However, HLA-E can also interact with the activating receptor CD94/NKG2C, thus leading to NK cell activation. These interactions are crucial during trophoblast implantation to abrogate NK cell lysis of semiallogeneic fetal tissue and, on the other hand, to activate NK cell functions in the process of tissue remodeling [11].

We have previously demonstrated that soluble (s)HLA-G concentration is higher in plasma samples from NB patients than in controls, and sHLA-G can be released by NB cells themselves, or by monocytes (stimulated by soluble factors secreted by tumor cells). Moreover, high sHLA-G plasma levels correlated with NB patients' relapse [4]. Finally, we have assessed that HLA-G is expressed by metastatic NB cells in the bone marrow from NB patients [6].

Also soluble HLA-E levels are higher in NB patients than in healthy controls. However, we have demonstrated that high plasma levels of sHLA-E at diagnosis correlated with a better overall survival (OS) of NB patients at follow-up, in contrast with sHLA-G [5].

Here, we demonstrated for the first time that sHLA-G and sHLA-E are present also in BM plasma samples derived from either NB patients at diagnosis or healthy donors. Moreover, we have assessed that SHLA-G and sHLA-E levels in BM plasma samples are related to the stage of the disease. Analysis of these patients at follow-up will reveal whether sHLA-G and sHLA-E concentration in BM plasma may predict the clinical outcome of NB patients.

\section{Materials and Methods}

2.1. Patients and Controls. The study was approved by the Ethics Committee of the G. Gaslini Institute, Genoa, Italy.
TABLE 2: Healthy donors' characteristics. First row indicates all the variables analyzed in healthy controls, second row indicates the subgroups for each variable, and third row indicates the number of subjects in each group.

\begin{tabular}{lcccc}
\hline \multicolumn{2}{c}{ Age (years) } & \multicolumn{2}{c}{ Sex } \\
Range & Mean \pm SD & M & F \\
\hline $20-54$ & $39.6 \pm 13$ & 8 & 5 \\
\hline
\end{tabular}

NB patients $(n=31)$ were diagnosed during 2016 in AIEOP centers. Bone marrow (BM) samples were collected at diagnosis and centralized at Istituto Giannina Gaslini in Genoa, Italy. BM plasma samples were obtained after centrifugation $\left(3000 \mathrm{~g} \times 10^{\prime}\right)$. NB patients were staged according to the International Neuroblastoma Staging System [12]. Patients' characteristics, that is, age at diagnosis, sex, MYCN amplification (single copy or amplified), BM infiltration, and stage, are summarized in Table 1.

As controls, BM aspirates were obtained from 13 healthy donors, selected according to the Transplant Unit Clinical Protocol of Ematologia 2 at the IRCCS San Martino-IST in Genoa, following a written informed consent at the time of donation. Samples were processed as described in [13], and an aliquot was taken at the end of processing to perform quality control tests, such as CD $34^{+}$cell count, in vitro progenitors' cell growth, and sterility. The remaining BM blood sample from this aliquot was subjected to centrifugation $(3000 g \times$ $10^{\prime}$ ) to obtain BM plasma. Donor's characteristics are summarized in Table 2.

All BM plasma samples were stored at $-80^{\circ} \mathrm{C}$ until use.

2.2. ELISA. Enzyme-Linked Immunosorbent Assay (ELISA) for sHLA-G and sHLA-E was performed as previously described [14]. Briefly, MaxiSorp Nunc-Immuno 96microwell plates (Nunc A/S, Roskilde, Denmark) were coated overnight at $4^{\circ} \mathrm{C}$ with $1 \mu \mathrm{g} / \mathrm{mL}$ of MEM-G9, specific for HLA-G HC (Exbio, Prague), that recognizes sHLAG1/G5, or 3D12 mAb, specific for HLA-E HC (eBioscience, Science Center Drive, San Diego, CA, USA). After three washes with PBS $0.05 \%$ Tween 20 (washing buffer), plates were saturated with $200 \mu \mathrm{L} / \mathrm{w}$ of PBS $2 \%$ BSA (Sigma, St. Louis, MO, USA) for $30 \mathrm{~min}$ at RT. One hundred $\mu \mathrm{L}$ of BM plasma samples and serial dilutions of 721.221.G1 cell line supernatant (for HLA-G) or total extract from normal peripheral blood mononuclear cells (standard) were added to each well and incubated at RT for 1 hour. After three washes, $100 \mu \mathrm{L}$ of detection reagent (HRP-conjugated anti- $\beta 2$ microglobulin $\mathrm{mAb}$, Exbio, Vestec, CZ) was added, 


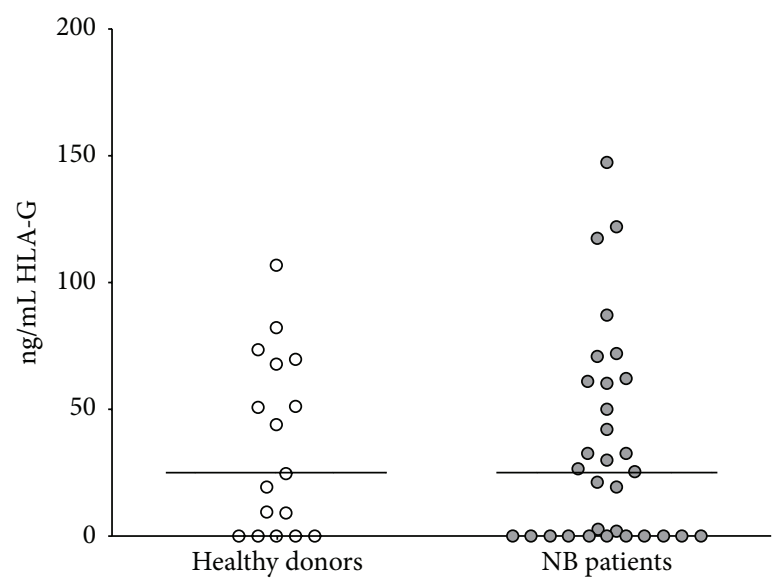

(a)

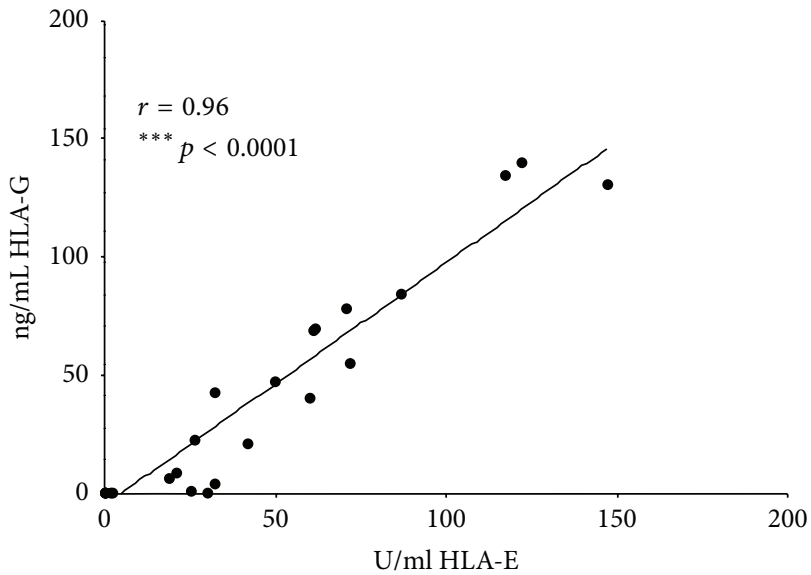

(c)

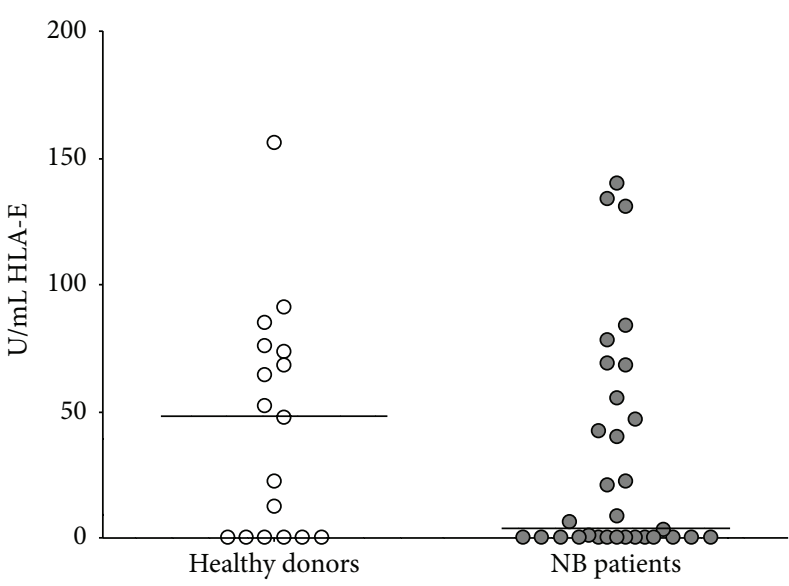

(b)

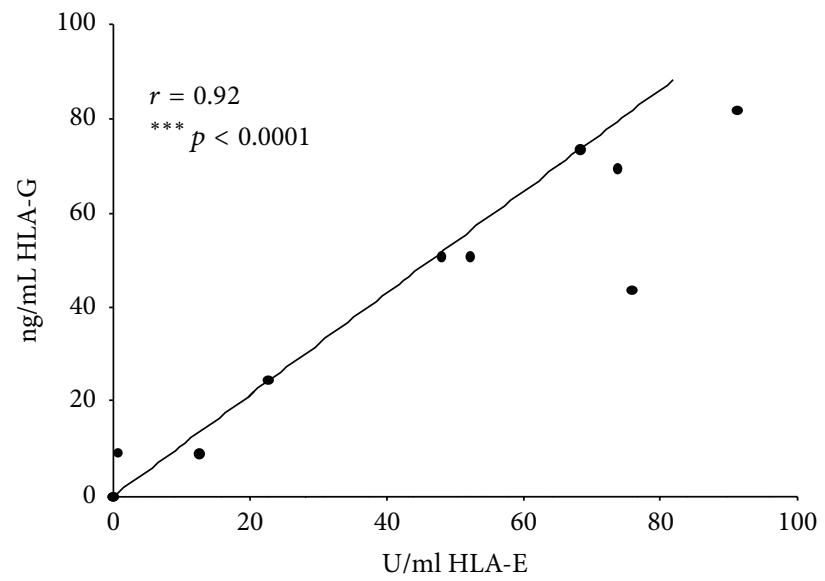

(d)

Figure 1: Levels of soluble (s)HLA-G (a) and sHLA-E (b) have been analyzed in BM plasma samples from NB patients (grey circles) and healthy BM donors (white circles). Horizontal lines indicated medians. Results are expressed as ng/ml (sHLA-G) or arbitrary units (U)/ml (sHLA-E). Correlation between BM plasma levels of sHLA-G and sHLA-E have been analyzed in NB patients (c) and controls (d). Linear regression of data and $r$ and $p$ values are indicated.

and plates were incubated for 1 hour at RT. After three washes, $100 \mu \mathrm{L}$ of TMB (substrate for HRP, Sigma) was added, and reaction was stopped after approximately 30 minutes by adding $\mathrm{H}_{2} \mathrm{SO}_{4} 5 \mathrm{~N}$. Absorbance at $450 \mathrm{~nm}$ was measured using Infinite ${ }^{\circledR} 200$ PRO spectrometer (Tecan Group Ltd., Seestrasse, Männedorf, Switzerland). Results are expressed as ng/mL sHLA-G and arbitrary units/mL sHLA-E (1 unit = quantity of sHLA-E in $1 \mu \mathrm{g}$ of total extract).

2.3. Statistics. Normal distribution of data was tested using Kolmogorov-Smirnov test, using Prism software (GraphPad Software Inc., La Jolla, CA). Since data distribution was not normal, differences in plasma levels between (i) patients and controls or (ii) different groups of patients were compared by Mann-Whitney test, using Prism software. Correlations between plasma levels of sHLA-G and sHLA-E were calculated by Spearman's test using Prism software. A $p$ value 0.05 was considered as statistically significant. Significance ranges are the following: ${ }^{*} p<0.05 ;{ }^{* *} p<0.01$; and ${ }^{* * *} p<0.001$.

\section{Results}

3.1. Soluble HLA-Ib Molecules Are Physiologically Present in BM Plasma Samples. First, we have tested sHLA-G and sHLA-E concentration in BM plasma samples from NB patients and healthy donors. As shown in Figure 1(a), sHLA$\mathrm{G}$ concentration was similar between NB patients (median \pm SE: $24.69 \pm 8.45 \mathrm{ng} / \mathrm{mL})$ and controls $(25.16 \pm 7.38 \mathrm{ng} / \mathrm{mL})$. In contrast, sHLA-E levels were lower in NB patients (3.72 \pm $7.89 \mathrm{U} / \mathrm{mL})$ than in controls $(48.01 \pm 10.93 \mathrm{U} / \mathrm{mL})$. However, such difference was not statistically significant, likely due to the wide distribution of the results in both groups (Figure 1(b)). Finally, sHLA-G and sHLA-E levels in BM plasma samples from NB patients $(r=0.96, p<0.0001$, Figure 1(c)) and healthy donors $(r=0.92, p<0.0001$, Figure $1(\mathrm{~d}))$ strongly correlated with each other.

3.2. Soluble HLA-Ib Molecules Correlated with Disease Progression. We have next analyzed possible correlation between sHLA-G and sHLA-E levels in BM plasma samples 


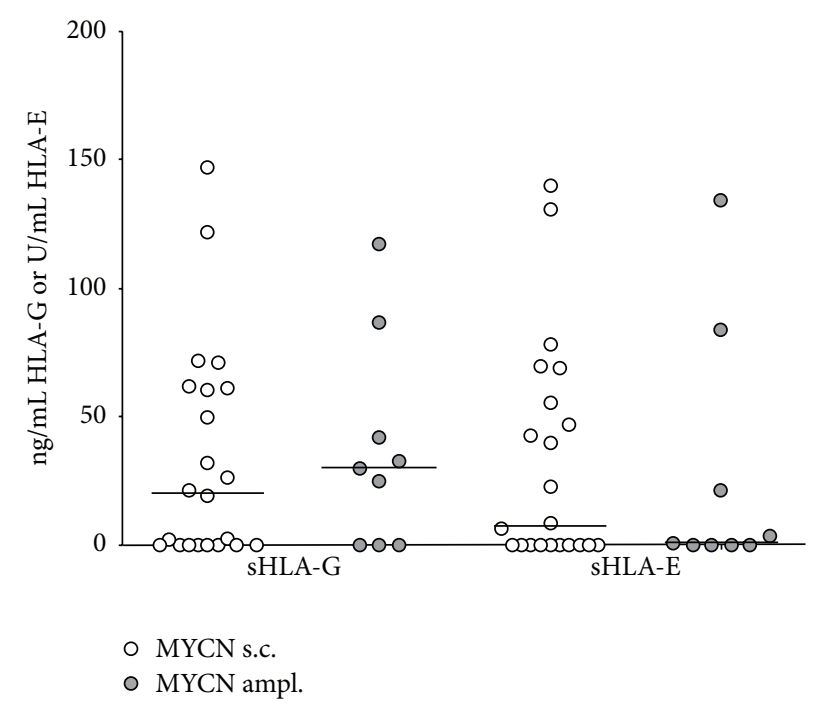

(a)

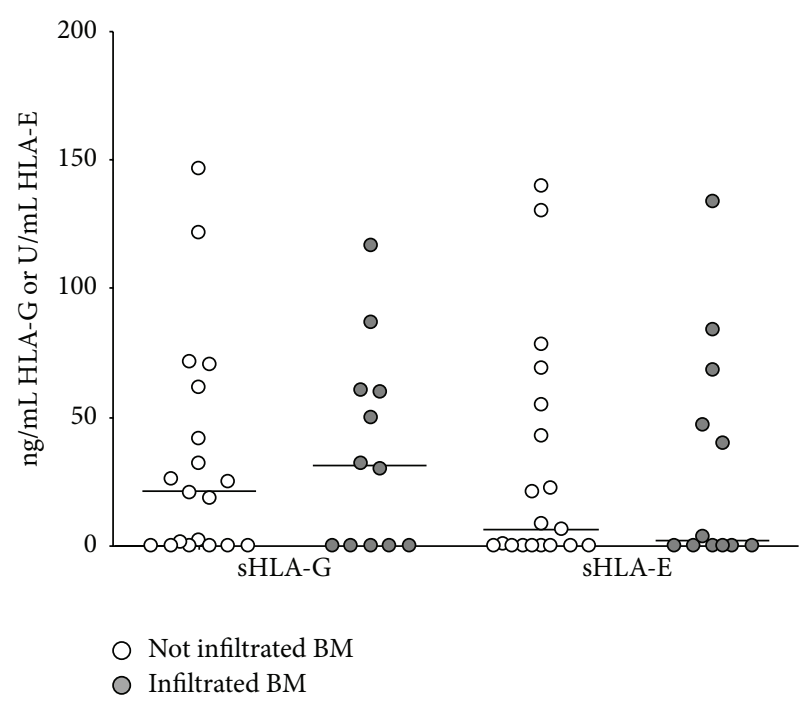

(b)

Figure 2: Levels of soluble (s)HLA-G and sHLA-E in BM plasma samples have been analyzed in patients presenting (grey circles) or not (white circles) MYCN amplification (a) or BM infiltration (b). Horizontal lines indicated medians. Results are expressed as ng/ml (sHLA-G) or arbitrary units (U)/ml (sHLA-E).

and patient's characteristics or clinical parameters. Accordingly, NB patients were divided into two groups on the basis of (i) MYCN amplification (single copy versus amplified), (ii) BM infiltration (not infiltrated versus infiltrated), (iii) age at diagnosis ( $<18$ months versus $>18$ months), (iv) stage of the disease (stages 1-2 versus stages 3-4), and (v) sex (male versus female). Next, differences in sHLA-G and sHLA-E levels between these groups of NB patients have been evaluated.

No significant differences in sHLA-G levels have been detected between NB patients (i) carrying amplified (29.96 $\pm 13.57 \mathrm{ng} / \mathrm{mL})$ or single-copy $(23.65 \pm 9.6 \mathrm{ng} / \mathrm{mL}) \mathrm{MYCN}$ gene (Figure 2(a)) and (ii) presenting $(31.17 \pm 11.99 \mathrm{ng} / \mathrm{mL})$ or not $(21.05 \pm 9.91 \mathrm{ng} / \mathrm{mL}) \mathrm{NB}$ cells infiltrating the BM (Figure 2(b)). In contrast, sHLA-E levels were higher in (i) patients with single-copy MYCN $(7.56 \pm 9.17 \mathrm{U} / \mathrm{mL})$ than in those with amplified MYCN $(1.03 \pm 16.2 \mathrm{U} / \mathrm{mL})$ (Figure $2(\mathrm{a})$ ) and in (ii) patients with infiltrated BM $(6.45 \pm 10.29 \mathrm{U} / \mathrm{mL})$ than in those without BM infiltration $(1.86 \pm 12.43 \mathrm{U} / \mathrm{mL})$ (Figure 2(b)). However, such differences were not statistically significant.

Furthermore, both sHLA-G and sHLA-E levels were similar between patients with an age below $(21.05 \pm 9.92 \mathrm{ng} / \mathrm{mL}$ sHLA-G and $6.45 \pm 10.29 \mathrm{U} / \mathrm{mL}$ sHLA-E) or above $(28.77$ $\pm 11.36 \mathrm{ng} / \mathrm{mL}$ sHLA-G and $2.37 \pm 12.81 \mathrm{U} / \mathrm{mL}$ sHLA-E) 18 months at diagnosis (Figure 3(a)). Notably, no correlation was found between age and sHLA-G or sHLA-E levels in BM plasma samples in healthy donors (data not shown).

Both sHLA-G and sHLA-E levels were significantly higher in patients with disease stages 3-4 (32.34 $\pm 8.08 \mathrm{ng} / \mathrm{mL}$ sHLA-G and $13.87 \pm 9.42 \mathrm{U} / \mathrm{mL}$ sHLA-E) than in those with disease stages $1-2(0 \pm 4.32 \mathrm{ng} / \mathrm{mL}$ sHLA-G and $0 \pm 3.27 \mathrm{U} / \mathrm{mL}$ sHLA-E, $p=0.01$ and 0.03 , resp.) (Figure 3(b)). Surprisingly, both sHLA-G and sHLA-E levels were found to be higher in male $(45.87 \pm 12.5 \mathrm{ng} / \mathrm{mL}$ sHLA-G and $34.19 \pm 14.83 \mathrm{U} / \mathrm{mL}$
sHLA-E) than in female $(2.52 \pm 8.81 \mathrm{ng} / \mathrm{mL}$ sHLA-G and 0 $\pm 8.18 \mathrm{U} / \mathrm{mL}$ sHLA-E, $p=0.05$ and 0.03 , resp.) NB patients (Figure 3(c)). In contrast, healthy donors showed higher levels of sHLA-G and sHLA-E in female $(50.74 \pm 14.1 \mathrm{ng} / \mathrm{mL}$ sHLA-G and $52.25 \pm 14.8 \mathrm{U} / \mathrm{mL}$ sHLA-E) than in male (12.35 $\pm 10.98 \mathrm{ng} / \mathrm{mL}$ sHLA-G and $11.33 \pm 13.25 \mathrm{U} / \mathrm{mL}$ sHLA-E) subjects. However, such differences were not statistically significant (Figure 3(d)).

\section{Discussion}

To the best of our knowledge, this is the first demonstration of the presence of sHLA-class Ib molecules HLA-G and HLA-E in BM plasma samples. Previous studies have demonstrated that sHLA-G can be released by some cell populations that are present in the BM environment, such as erythroblasts [15] and mesenchymal stromal cells [16-19]. In contrast, no information is available regarding HLA-E expression and release in the $\mathrm{BM}$. The strong correlation observed between the levels of these two molecules in BM samples either from $\mathrm{NB}$ patients or controls suggested that both molecules may be released by the same cell populations, or at least induced by similar stimuli.

We have previously demonstrated that metastatic NB cells in the BM expressed high levels of HLA-G on their surface, in contrast with primary tumors, that tested negative for HLA-G [6]. Here, we have demonstrated that both sHLA-G and sHLA-E are present at similar levels in NB patients and healthy donors, thus suggesting that malignant metastatic NB cells are unlikely involved in their release. This observation is further confirmed by the finding that BM infiltration by metastatic NB cells did not affect sHLA-G or sHLA-E levels in BM plasma samples. Moreover, MYCN amplification and age at diagnosis that represent important prognostic factors were 

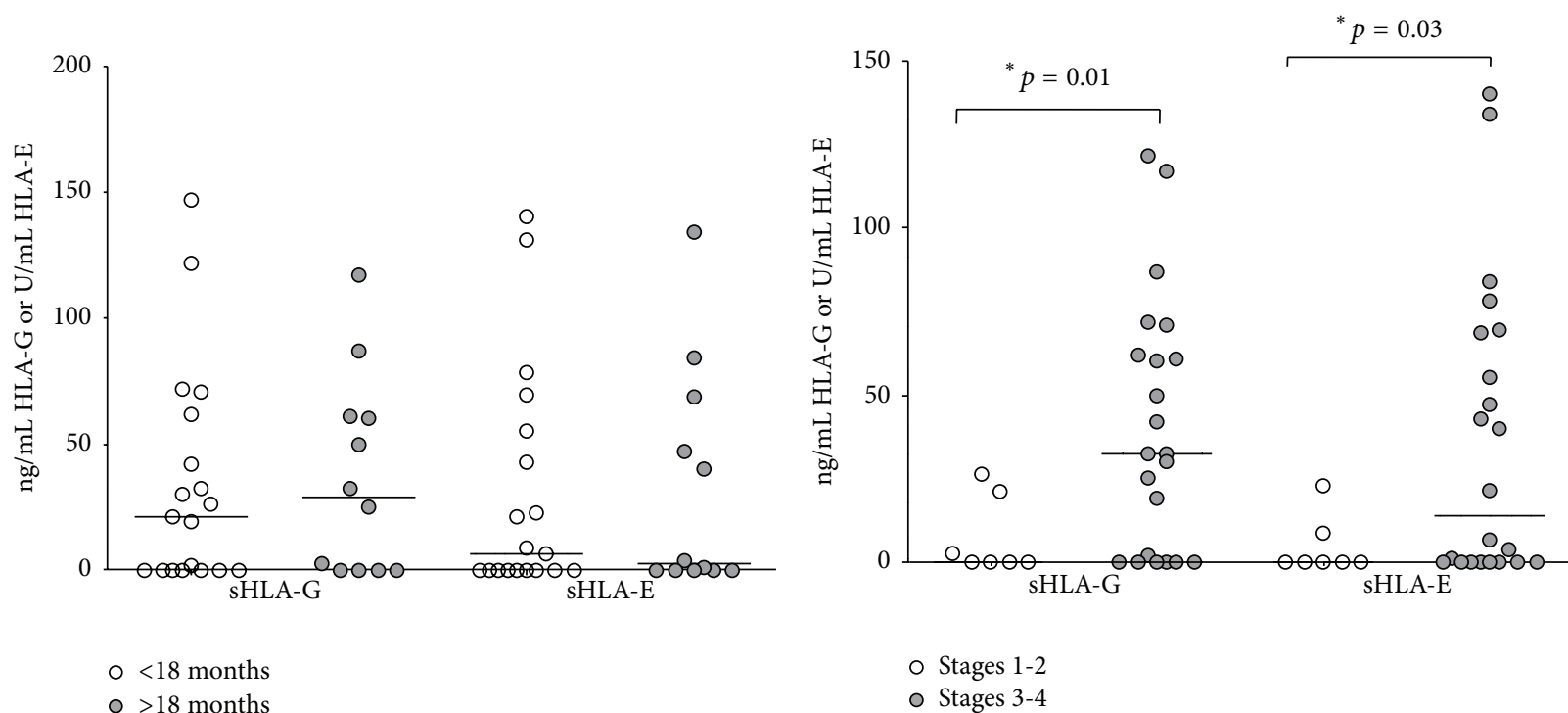

(a)
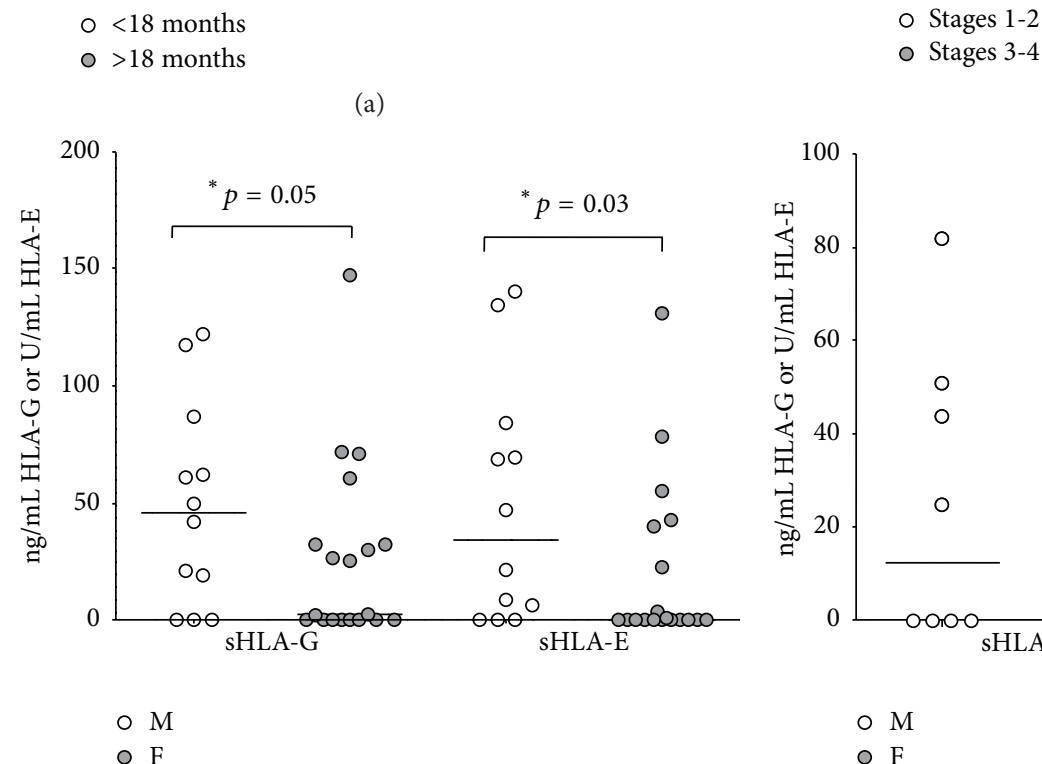

(c)

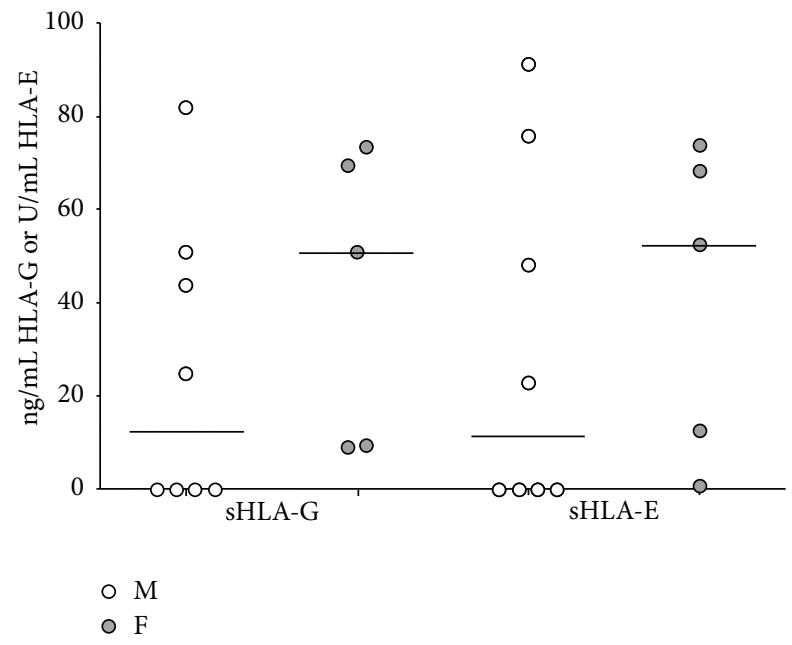

(d)

Figure 3: Levels of soluble (s)HLA-G and sHLA-E in BM plasma samples have been analyzed in patients with age at diagnosis above (grey circles) or below (white circles) 18 months (a) and in patients with stages 3-4 (grey circles) or 1-2 (white circles) disease (b). Differences between female (grey circles) or male (white circles) subjects have been analyzed in NB patients (c) and healthy donors (d). Horizontal lines indicated medians. Results are expressed as $\mathrm{ng} / \mathrm{ml}$ (sHLA-G) or arbitrary units (U)/ml (sHLA-E). $p$ values are indicated where differences are statistically significant.

not related to sHLA-G and sHLA-E levels in BM, thus further suggesting that these molecules might be released by $\mathrm{BM}$ stromal cells or BM resident cell populations instead of NB cells themselves, and may be present in the BM environment in physiological conditions. However, the increased tumor burden might be correlated to a higher release of tumorderived factor(s) that, in turn, can upregulate HLA-G and HLA-E production by BM stromal cells.

The finding that sHLA-G and sHLA-E BM plasma levels are higher in male than in female patients is in line with a previous study on multiple sclerosis, where the authors demonstrated that sHLA-G levels in plasma samples were higher in male than in female patients [20]. However, this study has been carried out using peripheral blood plasma samples, and this is the first demonstration of this difference between male and female subjects in bone marrow plasma samples. Notably, such difference may be a prerogative of NB patients, since sHLA-G and sHLA-E levels were higher in female than in male normal subjects.

The most important finding of our study is the demonstration that sHLA-G and sHLA-E levels were significantly higher in BM plasma samples from patients with metastatic disease than in patients with localized NB. This data may suggest that the levels of these molecules in the BM at diagnosis might be associated with disease progression and might be predictive of the clinical course of NB patients. However, this hypothesis can be confirmed only by analyzing the clinical parameters of these patients at follow-up. 


\section{Conclusions}

In conclusion, we demonstrated for the first time that soluble HLA-Ib molecules HLA-G and HLA-E are present in BM plasma samples in physiological and pathological conditions, and their concentration correlated with stage disease in NB patients. The prognostic value of sHLA-G and sHLA-E concentration in BM plasma samples from NB patients at diagnosis has to be confirmed in future studies.

\section{Competing Interests}

The authors declare that they have no competing interests.

\section{Authors' Contributions}

Vito Pistoia and Maria Valeria Corrias equally contributed as last author.

\section{Acknowledgments}

This study has been supported by Fondazione Italiana per la Lotta al Neuroblastoma and Ministero del Lavoro, della Salute e delle Politiche Sociali (Progetti di Ricerca Corrente-5 per mille). The authors thank Mrs. Camilla Valentino for excellent secretarial assistance. They also thank Dr. Sebastiano Barco and Dr. Giuliana Cangemi for their collaboration.

\section{References}

[1] N. R. Pinto, M. A. Applebaum, S. L. Volchenboum et al., "Advances in risk classification and treatment strategies for neuroblastoma," Journal of Clinical Oncology, vol. 33, no. 27, pp. 3008-3017, 2015.

[2] F. Morandi, M. V. Corrias, and V. Pistoia, "Evaluation of bone marrow as a metastatic site of human neuroblastoma," Annals of the New York Academy of Sciences, vol. 1335, no. 1, pp. 23-31, 2015.

[3] K. T. Vo, K. K. Matthay, J. Neuhaus et al., "Clinical, biologic, and prognostic differences on the basis of primary tumor site in neuroblastoma: a report from the International Neuroblastoma Risk Group project," Journal of Clinical Oncology, vol. 32, no. 28, pp. 3169-3176, 2014.

[4] F. Morandi, I. Levreri, P. Bocca et al., "Human neuroblastoma cells trigger an immunosuppressive program in monocytes by stimulating soluble HLA-G release," Cancer Research, vol. 67, no. 13, pp. 6433-6441, 2007.

[5] F. Morandi, G. Cangemi, S. Barco et al., "Plasma levels of soluble HLA-E and HLA-F at diagnosis may predict overall survival of neuroblastoma patients," BioMed Research International, vol. 2013, Article ID 956878, 9 pages, 2013.

[6] F. Morandi, P. Scaruffi, F. Gallo et al., "Bone marrow-infiltrating human neuroblastoma cells express high levels of calprotectin and HLA-g proteins," PLoS ONE, vol. 7, no. 1, article e29922, 2012.

[7] V. Pistoia, F. Morandi, G. Bianchi, A. Pezzolo, I. Prigione, and L. Raffaghello, "Immunosuppressive microenvironment in neuroblastoma," Frontiers in Oncology, vol. 3, article 167, 2013.

[8] D. T. Lau, M. D. Norris, G. M. Marshall, M. Haber, and L. J. Ashton, "HLA-G polymorphisms, genetic susceptibility, and clinical outcome in childhood neuroblastoma," Tissue Antigens, vol. 78, no. 6, pp. 421-427, 2011.

[9] P. Le Bouteiller and F. Lenfant, "Antigen-presenting function(s) of the non-classical HLA-E, -F and -G class I molecules: the beginning of a story," Research in Immunology, vol. 147, no. 5, pp. 301-313, 1996.

[10] V. Pistoia, F. Morandi, X. Wang, and S. Ferrone, "Soluble HLAG: are they clinically relevant?" Seminars in Cancer Biology, vol. 17, no. 6, pp. 469-479, 2007.

[11] F. Morandi and V. Pistoia, "Interactions between HLA-G and HLA-E in physiological and pathological conditions," Frontiers in Immunology, vol. 5, article 394, 2014.

[12] S. L. Cohn, A. D. J. Pearson, W. B. London et al., "The International Neuroblastoma Risk Group (INRG) classification system: an INRG task force report," Journal of Clinical Oncology, vol. 27, no. 2, pp. 289-297, 2009.

[13] D. Vicente, M. Podestà, A. Pitto et al., "Progenitor cells trapped in marrow filters can reduce GvHD and transplant mortality," Bone Marrow Transplantation, vol. 38, no. 2, pp. 111-117, 2006.

[14] F. Morandi, C. Venturi, R. Rizzo et al., "Intrathecal soluble HLAE correlates with disease activity in patients with multiple sclerosis and may cooperate with soluble HLA-G in the resolution of neuroinflammation," Journal of Neuroimmune Pharmacology, vol. 8, no. 4, pp. 944-955, 2013.

[15] C. Menier, M. Rabreau, J.-C. Challier, M. Le Discorde, E. D. Carosella, and N. Rouas-Freiss, "Erythroblasts secrete the nonclassical HLA-G molecule from primitive to definitive hematopoiesis," Blood, vol. 104, no. 10, pp. 3153-3160, 2004.

[16] F. Montespan, F. Deschaseaux, L. Sensébé, E. D. Carosella, and N. Rouas-Freiss, "Osteodifferentiated mesenchymal stem cells from bone marrow and adipose tissue express HLA-G and display immunomodulatory properties in Hla-mismatched settings: implications in bone repair therapy," Journal of Immunology Research, vol. 2014, Article ID 230346, 10 pages, 2014.

[17] F. Morandi, L. Raffaghello, G. Bianchi et al., "Immunogenicity of human mesenchymal stem cells in HLA-class I-restricted Tcell responses against viral or tumor-associated antigens," Stem Cells, vol. 26, no. 5, pp. 1275-1287, 2008.

[18] R. Rizzo, D. Campioni, M. Stignani et al., "A functional role for soluble HLA-G antigens in immune modulation mediated by mesenchymal stromal cells," Cytotherapy, vol. 10, no. 4, pp. 364$375,2008$.

[19] Z. Selmani, A. Naji, I. Zidi et al., "Human leukocyte antigenG5 secretion by human mesenchymal stem cells is required to suppress T lymphocyte and natural killer function and to induce CD4+ CD25highFOXP3+ regulatory T cells," Stem Cells, vol. 26, no. 1, pp. 212-222, 2008.

[20] F. Alsahebfosoul, A. Zavaran Hosseini, R. Salehi, M. Etemadifar, N. Esmaeil, and A. Jamshidian, "Evaluation of soluble human leukocyte antigen-G (sHLA-G) isoforms and regulatory T cells in relapsing-remitting multiple sclerosis," Iranian Journal of Allergy, Asthma and Immunology, vol. 14, no. 3, pp. 298-305, 2015. 


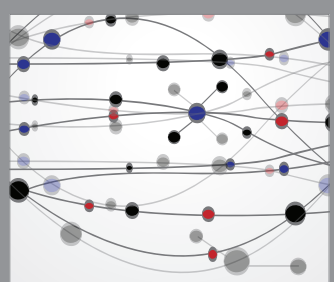

The Scientific World Journal
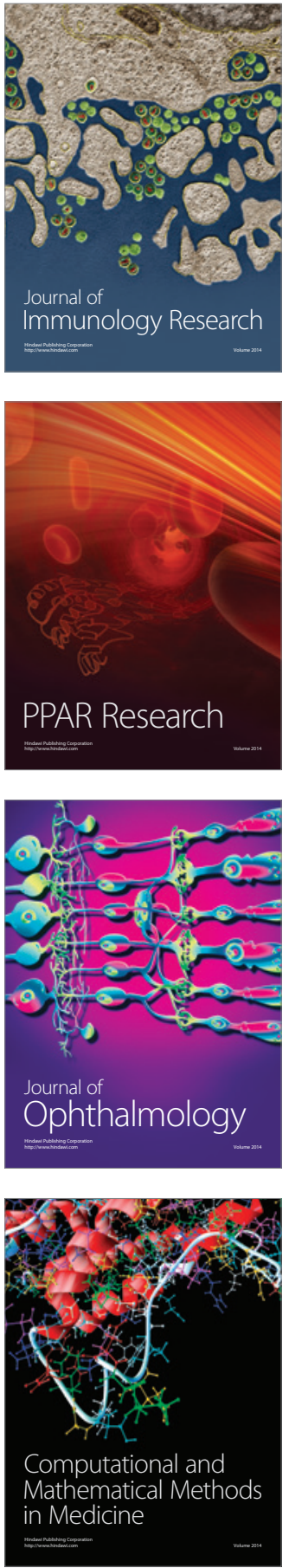

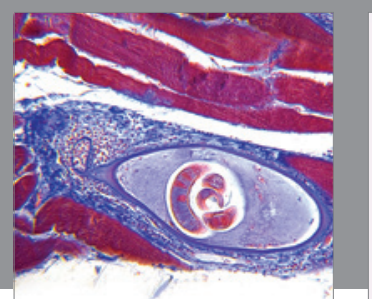

Gastroenterology Research and Practice

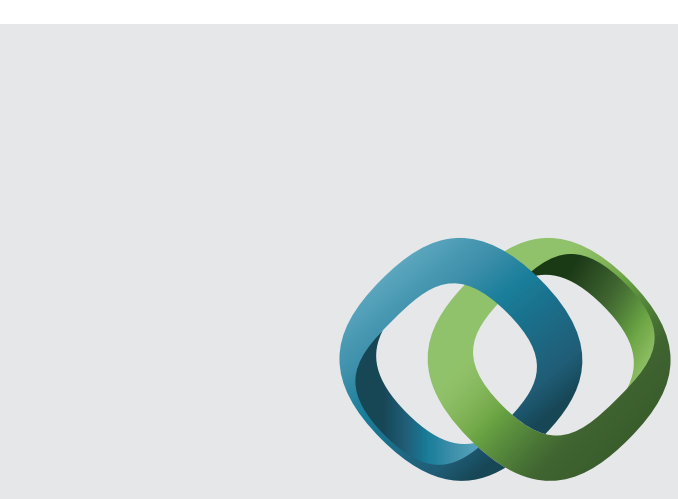

\section{Hindawi}

Submit your manuscripts at

http://www.hindawi.com
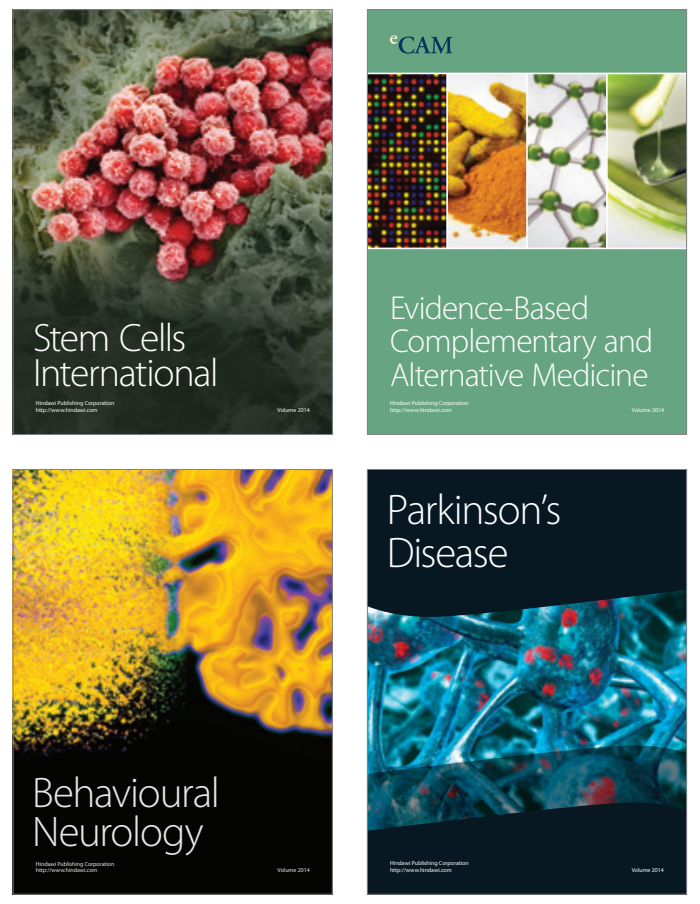
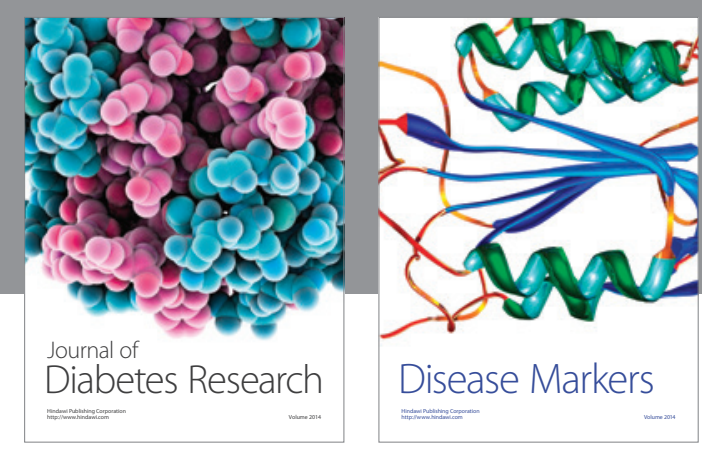

Disease Markers
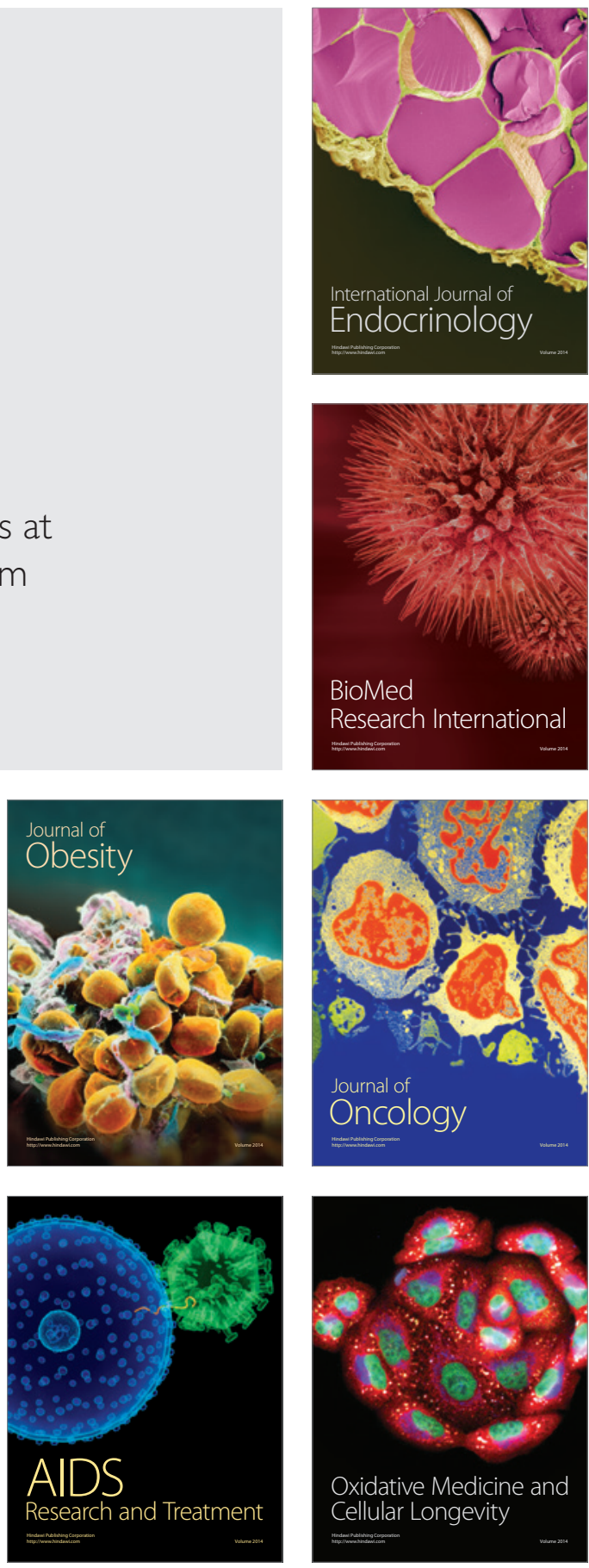